\title{
A Magnetically Steered Endolaser Probe for Automated Panretinal Photocoagulation
}

\section{Journal Article}

\section{Author(s):}

Charreyron, Samuel (D); Gabbi, Edoardo; Boehler, Quentin (D); Becker, Matthias; Nelson, Bradley J.

Publication date:

2019-04

\section{Permanent link:}

https://doi.org/10.3929/ethz-b-000334952

Rights / license:

In Copyright - Non-Commercial Use Permitted

Originally published in:

IEEE Robotics and Automation Letters 4(2), https://doi.org/10.1109/LRA.2018.2888894

\section{Funding acknowledgement:}

165564 - Soft Magnetic Robots: Modeling, Design and Control of Magnetically Guided Continuum Manipulators (SNF) 743217 - Soft Micro Robotics (EC) 


\title{
A Magnetically Steered Endolaser Probe for Automated Panretinal Photocoagulation
}

\author{
Samuel L. Charreyron ${ }^{1}$, Edoardo Gabbi, Quentin Boehler ${ }^{1}$, Matthias Becker ${ }^{2}$, and Bradley J. Nelson ${ }^{1}$
}

\begin{abstract}
The advanced state of diabetic retinopathy, a leading cause of blindness, is treated by panretinal photocoagulation (PRP), a repetitive procedure performed by a surgeon using a handheld laser probe. In its place we propose a soft-robotic flexible probe precisely steered using magnetic fields generated by an external magnetic steering system. We develop a kinematic model for the PRP task and show that the process can be automated given image feedback of the retina through a fundus camera. We demonstrate the concept in an eye phantom of a human eye, achieving clinical-level accuracy and faster speeds than human surgeons.
\end{abstract}

Index Terms-Surgical Robotics: Steerable Catheters/Needles; Automation in Life Sciences: Biotechnology, Pharmaceutical and Health Care

\section{INTRODUCTION}

D IABETES mellitus is a disease affecting 285 million adults worldwide. Diabetic retinopathy (DR) involves damage to the retina as a consequence of high blood sugar. proliferative diabetic retinopathy (PDR), the advanced state of DR, leads to vision loss. It is a leading cause of blindness with one third of diabetics showing some form of DR [1]. The prevalence of diabetes and DR is projected to rapidly increase with an aging population.

For several decades, the gold standard of treatment for PDR has been PRP. A recent survey of ophthalmic surgeons has shown that PRP is unanimously used in treating patients with high-risk PDR [2]. PRP consists of delivering several thousand evenly spaced laser burns throughout the retina. This helps improve oxygen supply to the inner retina and, in most cases, stops neovascularization [3]. Depending on the patient's discomfort, PRP is performed in one or more sessions lasting approximately twenty minutes. It may be repeated after several months if symptoms do not subside. PRP is often performed as an outpatient procedure using a non-invasive slit-lamp laser. However, in advanced cases of PDR vitreoretinal surgery is often required due to blood leakage or retinal detachment. In these cases PRP is performed in an operating room using a handheld endolaser tool inserted into the ocular cavity through the pars plana.

Manuscript received: September, 10, 2018; Revised November, 21, 2018; Accepted December, 15, 2018.

This paper was recommended for publication by Editor Pietro Valdastri upon evaluation of the Associate Editor and Reviewers' comments. *This work was partially funded by the Swiss National Science Foundation gran 200021_165564 and the ERC Advanced Grant 743217, Soft Micro Robotics (SOMBOT).

${ }^{1}$ Multi Scale Robotics Lab, ETH Zurich, Tannenstrasse 3, 8092 Zurich, Switzerland samuelch@ethz.ch

${ }^{2}$ Stadtspital Triemli, Birmensdorferstrasse 497, 8003 Zurich, Switzerland

Digital Object Identifier (DOI): see top of this page.

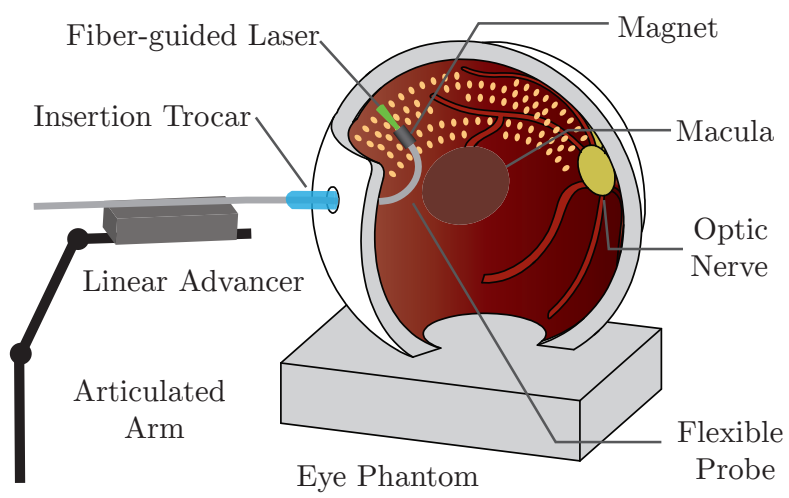

Fig. 1. Schematic of endolaser photocoagulation using a magnetically steered endolaser probe in an open-sky model of the eye.

More recently, slit-lamp pattern scanning lasers have partially automated the procedure, allowing the surgeon to apply a number of preplanned points in rapid succession. Pattern scanning lasers have reduced both the time needed for a procedure and patient discomfort [4]. Endolaser PRP, however, is notoriously difficult for less experienced surgeons, painstakingly repetitive and tedious, and uncomfortable for the patient and the surgeon [5]. As such, endolaser PRP presents itself as an excellent candidate for robotic surgery with potential for partial or full automation.

Pattern scanning lasers allow the surgeon to preselect a desired array of target points on a fundus image of the retina and deliver burns to these points in rapid succession. In robotics research, the handheld Micron Surgical Robot [6] has been used to demonstrate automatic placement of laser spots using visual servoing. The device was recently demonstrated in wet eye phantoms and porcine eyes [7]. Nevertheless, the drawback of such a system is the limited working area of diameter $2 \mathrm{~mm}$, requiring the operator to hold the tool at a number of positions in order to cover the entire retina. While automatic navigation of the Micron could decrease the operation time, the tool would still need be held by the surgeon. The surgeon, therefore, must remain relatively still for long periods with a rigid tool, increasing risk of damage to the sensitive structures of the eye.

Magnetic steering of rods or catheters, which has seen clinical adoption for cardiology applications with the Stereotaxis robotic magnetic navigation system [8], is well-suited for steering small-diameter devices in confined environments [9] including the inner cavity of the eye [10].

In this work, we introduce a new automatic laser photocoagulation system using magnetic steering. Through a 
single pars plana insertion, the magnetically steered endolaser is able to reach a large area on the retina. Laser spot placement is automated using a kinematic model of the probe and visual servoing based on the probe model. The tool is demonstrated in a 3D-printed dry phantom of the posterior eye on an array of 273 regularly spaced points. We show that the laser spot is accurately steered over the entire workspace. A key advantage of this approach is that it reaches points faster than that reported in related work or estimated for manual procedures.

The paper is organized as follows. We first describe the components that comprise our system in II. In III, we outline mathematical models describing the kinematics of our system and explain how uncertain parameters were calibrated. In IV, we outline the control strategy that was used to steer the laser spot to desired positions. Finally in V, we report results from an experiment in an eye phantom and discuss automation of PRP.

\section{SYSTEM OVERVIEW}

\section{A. Principle of Operation}

Conventionally, a handheld endolaser probe is manipulated through several pars-plana trocars that are inserted through the sclera into the intraocular space during a vitrectomy. The surgeon must switch trocars and hands to access the entire retina, and several straight-tip or curved probes may be used to facilitate access [5]. In contrast, we propose a flexible endolaser probe consisting of a long flexible shaft and a magnetic distal segment, inserted through a single trocar, with access to the entire retina. The endolaser probe is bent using magnetically-generated torques at the tip, while the length of the probe is controlled by a linear advancer unit providing three degrees-of-freedom (DOF) for control. Similar to conventional endolasers, an optical fiber guides laser illumination to the distal tip of the probe, which is projected onto the retina. The laser light projected on the retina is viewed using a monoscopic fundus camera view. The entire device can be interfaced with a computer allowing for full-automation of the procedure.

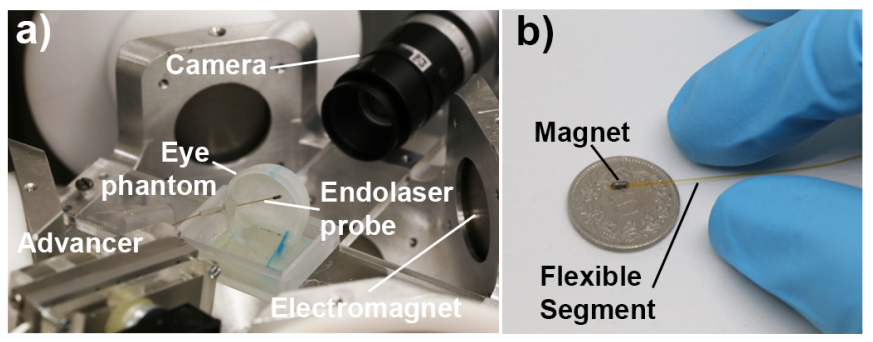

Fig. 2. a) Photo showing the experimental setup used in this work. On the bottom left, the linear positioner is attached to an articulated arm and controls the insertion of the endolaser probe. The setup fits inside the electromagnets of the OctoMag. At the top right, a camera provides an image of the back of the eye phantom. b) Photo showing the endolaser probe with a permanent magnet at the distal end. Swiss 10 rappen coin (diameter $19 \mathrm{~mm}$ ) shown for scale.

A conceptual illustration of the system operation is shown in Fig. 1 and pictures of the experimental setup and probe are shown in Fig. 2.

\section{B. Magnetic Endolaser Probe}

The endolaser probe consists of a fiber optic cannula (Doric Lenses) with core diameter $100 \mu \mathrm{m}$ and outer diameter (OD) $125 \mu \mathrm{m}$. The optical fiber was placed inside a polyimide tube with dimensions $177 \mu \mathrm{m}$ (ID), $215 \mu \mathrm{m}$ (OD). A NdFeB $2 \mathrm{~mm}$ long ring magnet $450 \mu \mathrm{m}$ (ID), $850 \mu \mathrm{m}$ (OD) was glued at the distal end of the probe. Finally, the optical fiber was placed inside a custom-designed adaptor for a FC/PC connection.

\section{Magnetic Steering System}

Magnetic fields used to steer the endolaser probe were generated by the OctoMag [11], an eight-electromagnet magnetic steering system with a cubic workspace of side $10 \mathrm{~cm}$. Magnetic fields were generated with a flux density $\mathbf{B}$ magnitude up to $60 \mathrm{mT}$ inside the workspace resulting in torques $\mathbf{T}_{m}$ applied to the distal magnet of magnetic moment $\mathbf{m}$ of the endolaser according to (1).

$$
\mathbf{T}_{m}=\mathbf{m} \times \mathbf{B}
$$

\section{Linear Advancer}

The endolaser probe was attached to an SLC 2490 (SmarAct) piezo-actuated linear positioner with a position accuracy of $1 \mu \mathrm{m}$. The positioner was mounted on a lockable arm with two DOF, allowing flexible access to the eye phantom. The positioner was used to insert and retract the probe from the pars-plana trocar on the eye phantom.

\section{E. Laser}

For safety and simplicity, a low power laser source was used for this work rather than the high power $522 \mathrm{~nm}$ lasers customarily used for photocoagulation. In the future, one could readily substitute the lower power laser with a clinical laser without change to the probe design. A fiber-coupled near infrared (NIR) $780 \mathrm{~nm}$ diode laser with a maximum optical power of $12 \mathrm{~mW}$ was driven using an automatic current controller (Thorlabs). The laser output was modulated using the current controller's analog input through an SMA connection, and a serial computer interface to the laser was created using an Arduino Due development board.

\section{F. Eye Phantom}

An open-sky semi-transparent dry eye phantom was used so that the laser spots could be tracked by a NIR camera throughout the entire posterior region without using a wide-angle viewing system. A spherical eyeball of diameter $24 \mathrm{~mm}$ was 3D printed, corresponding to average adult anatomical data reported in the literature [12]. A fixed pars-plana sclera insertion trocar with a known position was added through which the endolaser could be inserted and retracted. 


\section{G. Imaging}

Image feedback was provided through a Basler Aca2040-90um camera with a resolution of $2048 \times 2048$ pixels and sensitive to NIR light. The camera was equipped with a $25 \mathrm{~mm}$ lens (Fujinon) and a $5 \mathrm{~mm}$ extension ring, allowing a field of view of approximately $30 \mathrm{~mm}$ at a working distance of $95 \mathrm{~mm}$. The camera was operated at its maximum framerate of 50 frames-per-second (FPS). A view of the back camera is shown in Fig. 3 a).

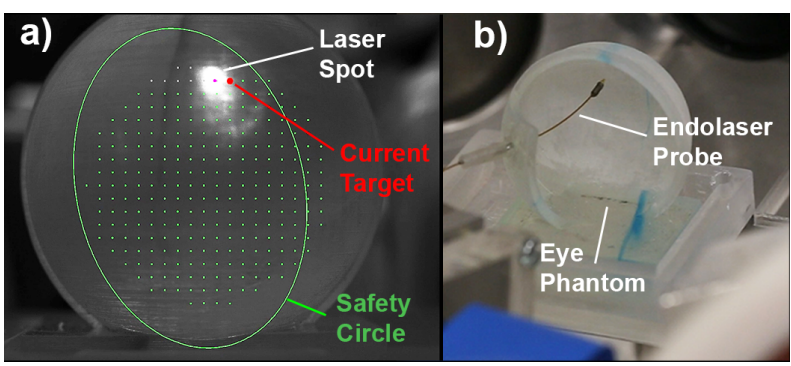

Fig. 3. a) View from the back camera. The grey/green points indicate targets that are not yet achieved/pending respectively. The red circle indicates the current target point, the green ellipse shows the circle of safety, and the pink dot shows the tracked laser spot position. The white blob is the laser spot. b) Front view of the probe for the same configuration.

\section{H. Image Processing}

The laser spot on the eye phantom was tracked in real-time using a simple image processing pipeline. A binary threshold was applied to the image to segment the bright laser spot from the background. Contour detection and filtering based on the contour size were used to detect the blob formed by the laser spot. The contour's centroid was used as the 2D tracked laser spot position. All image processing steps were performed using OpenCV [13].

\section{MODELING}

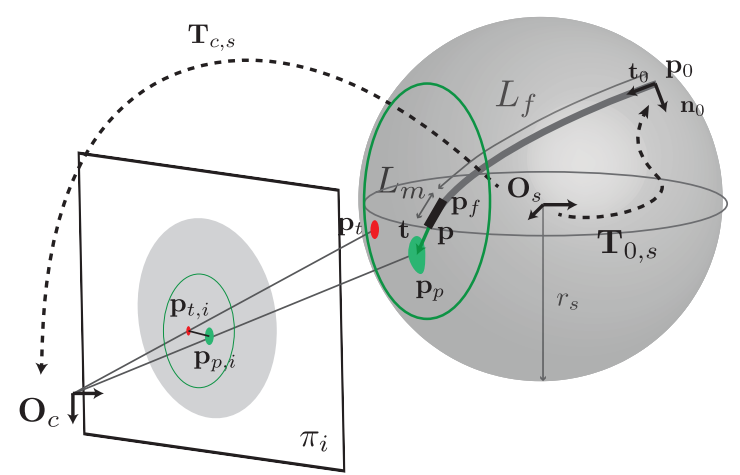

Fig. 4. Diagram illustrating the model used in our work. The eye is modeled as a sphere centered at $\mathbf{O}_{s}$. The flexible probe's centerline is represented by the dark gray arc and is inserted through the fixed proximal point $\mathbf{p}_{0}$. The probe's proximal orientation is defined by proximal tangent $\mathbf{t}_{0}$ and normal $\mathbf{n}_{0}$ vectors. At the distal end, the projected laser spot $\mathbf{p}_{p}$ is shown as the green blob, and the target position $\mathbf{p}_{t}$ is represented as the red circle. The scene is observed by a camera at $\mathbf{O}_{c}$ and projected onto the image plane $\pi_{i}$. The circle of safety is shown as the green oval.
TABLE I

PARAMETERS USED FOR MECHANICAL MOdel OF ENDOlaSer

\begin{tabular}{|c|c|c|c|c|}
\hline \multicolumn{3}{|c|}{ Magnet } & \multicolumn{2}{|c|}{ Flexible Shaft } \\
\hline Mass & Remanence & Length & Density & $\begin{array}{l}\text { Young's } \\
\text { Modulus }\end{array}$ \\
\hline $6 \mu \mathrm{g}$ & $1.09 \mathrm{~T}$ & $2 \mathrm{~mm}$ & $1.25 \mathrm{~kg}$ & $12.6 \mathrm{GPa}$ \\
\hline
\end{tabular}

\section{A. Endolaser Kinematics}

The endolaser probe was modeled as a magnetic continuum device with a variable length isotropic flexible segment corresponding to the shaft of length $L_{f}$ and a distal rigid magnetic segment $L_{m}$ corresponding to the distal magnet. The total length is $L=L_{f}+L_{m}$. For simple magnetic continuum devices with a single magnetic segment, neglecting the effect of gravity, one can model the device using a constant curvature model [14], where the curvature is a function of the applied magnetic field and shaft length. The bending characteristics can be analyzed in a single manipulation plane. The manipulation plane is spanned by the proximal tangent vector $\mathbf{t}_{0}$ and the proximal normal vector $\mathbf{n}_{0}$. These are shown in Fig. 4. The arc describing the continuum is fully described by three scalars: the curvature $k$, the flexible segment length $L_{f}$, and an angle $\theta$ describing the rotation of the manipulation plane about the proximal tangent. Without loss of generality, we define a canonical vector $\hat{\mathbf{n}}_{0}$ normal to the proximal tangent and define $\mathbf{n}_{0}$ as

$$
\mathbf{n}_{0}=\mathbf{R}\left(\hat{\mathbf{n}}_{0}, \theta\right) \hat{\mathbf{n}}_{0}
$$

where the rotation matrix is expressed using the Euler-Rodrigues formula where $[\cdot]_{\times}$represents the skew-symmetric matrix associated with a vector.

$$
\mathbf{R}(\mathbf{v}, \theta)=\mathbf{I}_{3 \times 3}+\sin \theta[\mathbf{v}]_{\times}+(1-\cos \theta)[\mathbf{v}]_{\times}^{2}
$$

Following this point we regroup the kinematic variables into a vector $\mathbf{x}=\left[\begin{array}{lll}k & L & \theta\end{array}\right]^{T}$ for convenience.

The equations governing the position at the end of the flexible segment $\mathbf{p}_{f}$ and the tangent vector $\mathbf{t}$ are as follows

$$
\begin{aligned}
\mathbf{p}_{f}(\mathbf{x}) & =\frac{1}{k}\left[(1-\cos \varphi) \mathbf{t}_{0}+\sin \varphi \mathbf{n}_{0}\right] \\
\mathbf{t}(\mathbf{x}) & =\sin \varphi \mathbf{t}_{0}+\cos \varphi \mathbf{n}_{0}
\end{aligned}
$$

where $\varphi=k L_{f}$. The distal position at the end of the magnet $\mathbf{p}$ is

$$
\mathbf{p}(\mathbf{x})=\mathbf{p}_{f}(\mathbf{x})+L_{m} \mathbf{t}(\mathbf{x})
$$

From [14], the relationship between the curvature, length, and magnetic field is given by the following equation

$$
k E I-M B_{0} \sin (\gamma-\varphi)=0
$$

where $E$ is the Young's modulus, $I$ the second moment of area of the flexible segment, $B_{0}$ the magnetic field magnitude, $\gamma$ the angle between the magnetic field and $\mathbf{t}_{0}$ and $M$ the magnitude of the distal magnet's magnetic moment.

The mechanical properties of the probe are shown in Table I. The mass of the magnet and tubes were measured with an analytic scale, while the magnet remanence was measured 
with a vibrating sample magnetometer. The Young's modulus of the flexible segment $E^{\text {cal }}$ was calibrated using nonlinear least-squares from visual data using the Ceres solver [15] as shown in (8). A calibrated camera was modeled using a pinhole camera model, resulting in perspective projection model $P_{c}$ relating $3 \mathrm{D}$ positions to image positions. At a configuration $j$, length $L$ was fixed and a magnetic field was rotated in a plane parallel to the image plane, resulting in currents $\mathbf{I}^{j}$, modeled distal position $\mathbf{p}^{j}$, and the observed distal position in the image $\mathbf{y}_{i}^{j}$ was recorded. The average position error in the calibration was $174 \mu \mathrm{m}$. Two examples are shown in Fig. 5.

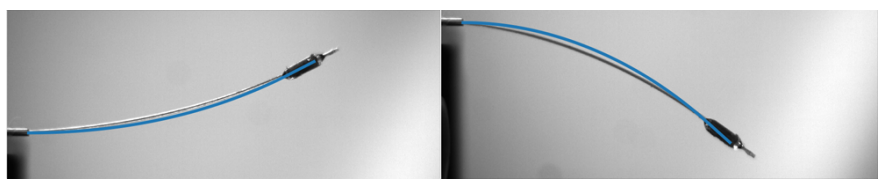

Fig. 5. Sample images from the dataset used for calibrating the flexible segment's Young's modulus. The calibrated model positions are shown as the blue line over the image.

$$
E^{c a l}=\arg \min _{E} \sum_{j}\left\|P_{c}\left(\mathbf{p}^{j}\left(L, \mathbf{I}^{j}, E\right)\right)-\mathbf{y}_{i}^{j}\right\|^{2}
$$

\section{B. Laser Projection}

The eye phantom was modeled as a sphere with center $\mathbf{O}_{s}$ and radius $r_{s}$. The laser-guiding fiber was assumed to coincide with the distal tangent of the endolaser, and the laser was modeled as a single ray emanating from the distal position $\mathbf{p}$ and along the distal tangent $\mathbf{t}$. The laser spot center on the eye model $\mathbf{p}_{P}$ is obtained by solving a quadratic with the following discriminant.

$$
\Delta=\left(\mathbf{t} .\left(\mathbf{p}-\mathbf{O}_{s}\right)\right)^{2}-\left\|\mathbf{p}-\mathbf{O}_{s}\right\|^{2}+r_{S}^{2}
$$

where $\Delta>0$ and the laser ray intersects the sphere in two points along distances $d_{1,2}$ along the line passing through $\mathbf{p}(L)$ and parallel to $\mathbf{t}(L)$. We choose the point projected in the forward direction corresponding to the single positive distance $d_{1,2}$ resulting in the projected point $\mathbf{p}_{p}$.

$$
\begin{aligned}
d_{1,2}(\mathbf{x}) & =-\mathbf{t} \cdot\left(\mathbf{p}-\mathbf{O}_{s}\right) \pm \sqrt{\Delta} \\
d & =\max \left\{d_{1}, d_{2}\right\} \\
\mathbf{p}_{p}(\mathbf{x}) & =\mathbf{p}(\mathbf{x})+d(\mathbf{x}) \mathbf{t}(\mathbf{x})
\end{aligned}
$$

\section{Camera Projection}

The camera intrinsic model comprised a pinhole camera projection model with radial tangent distortion. Intrinsic parameter calibration was performed using a chessboard grid with OpenCV [13].

The camera was registered extrinsically to the coordinate frame of the eye model using a chessboard pattern with positions known in the eye model frame. The transformation $T_{c, s}$ transforming from the sphere frame to the camera frame was computed using OpenCV's solvePnP function. Therefore, for a position $\mathbf{p}$ expressed in the sphere frame, its projected position in the image $\mathbf{p}_{i}$ is represented by the distortion and pinhole projection.

$$
\mathbf{p}_{i}=P_{c}\left(T_{c, s} \mathbf{p}\right)
$$

Given a calibrated camera with intrinsic matrix $\mathbf{K}_{i}$, it is possible to map image coordinates to $3 \mathrm{D}$ positions on the sphere. The ray from the camera center to the undistorted image point $\tilde{\mathbf{p}}_{i}$ is

$$
\mathbf{v}=\mathbf{K}_{i}^{-1}\left[\begin{array}{ll}
\tilde{\mathbf{p}}_{i} & 1
\end{array}\right]^{T}
$$

We can directly use (9), replacing $\mathbf{p}$ with the camera position and $\mathbf{t}$ with $\mathbf{R}_{s, c} \mathbf{v}$. We also modify (10) to use the minimum instead of maximum.

\section{Endolaser Proximal Pose}

The proximal position $\mathbf{p}_{0}$ and orientation is assumed to be fixed. The proximal position is known from the computer model of the eye phantom. A simple procedure was followed to calibrate the proximal tangent vector $\mathbf{t}_{0}$. The laser was retracted to its insertion, and its projection point on the image was recorded. Using (14) and the proximal position, the tangent direction at the insertion point was calculated.

\section{E. Magnetic Model}

The mapping between the electromagnets and the magnet fields inside the workspace is expressed using a calibrated dipole-based model [16], where $\mathbf{A}(\mathbf{p})$ is the position dependent $3 \times 8$ actuation matrix.

$$
\mathbf{B}(\mathbf{p})=\mathbf{A}(\mathbf{p}) \mathbf{I}
$$

One can find a set of currents $\mathbf{I}$ resulting in a desired magnetic field at a position using the Moore-Penrose pseudoinverse $\mathbf{A}^{\dagger}(\mathbf{p})$.

$$
\mathbf{I}=\mathbf{A}^{\dagger}(\mathbf{p}) \mathbf{B}(\mathbf{p})
$$

For simplicity, due to the field homogeneity, we assumed the position of the magnet to be at the center of the workspace for the purpose of the magnetic calculations. Over the range of movement, the field magnitude was estimated to vary by at most $4 \mathrm{mT}$ in magnitude and $4^{\circ}$ in angle, which would result in small changes in the catheter's configuration.

\section{F. Circle of Safety}

Given knowledge of the length of the probe $L$, the radius of the sphere $r_{s}$, and the insertion point $\mathbf{p}_{0}$, one can define a region of safety in which the probe will not come into contact with the sphere no matter how much it is deflected. This region is defined by the intersection of a sphere centered at $\mathbf{p}_{0}$ and of radius $L$ and the sphere representing the eye. The intersection of two spheres is a circle. Therefore, there will be no contact provided the endolaser remains inside the sphere cap delimited by this circle. We project this circle onto the image using (13) and use it as a visual representation for the area of the workspace that is safe to operate in. This circle is represented in green in Fig. 4 and is visible in the video submission. 


\section{CONTROL}

\section{A. Inverse Kinematics}

To perform feedforward control on changes in the target position $\mathbf{p}_{t}$, we used inverse kinematics (IK) of the model. Finding a set of control inputs $\mathbf{x}_{f}=\left[\begin{array}{lll}k & L_{f} & \theta\end{array}\right]^{T}$ resulting in a desired point projected on the sphere was expressed as solving the following nonlinear program.

$$
\begin{array}{ll}
\underset{\mathbf{x}}{\operatorname{minimize}} & \left\|\mathbf{p}_{p}(\mathbf{x})-\mathbf{p}_{t}\right\|^{2} \\
\text { subject to } & d_{\min } \leq d(\mathbf{x}) \leq d_{\max } \\
& \left\|\mathbf{p}(\mathbf{x})-\mathbf{p}_{s}\right\|^{2} \leq r_{s}^{2}
\end{array}
$$

Minimizing (17) is underconstrained since we can control three DOF. We therefore added constraints on the distance between the distal tip and the projected point on the retina. Rather than perform damped-least squares, which usually works without constraints or with constraints added as a penalizing term in the objective, we formulated the IK problem as a constrained nonlinear program. In order to ensure that the endolaser tool always remains inside the sphere, we constrained the distal position to remain inside the sphere using (19).

The nonlinear program was solved using the SLSQP [17] nonlinear optimization function of the SciPy library [18]. The limits on the tip distance to the sphere $d_{\min }$ and $d_{\max }$ were set to $1 \mathrm{~mm}$ and $3 \mathrm{~mm}$, respectively.

\section{B. Analytical Jacobians}

While the nonlinear program in (17) can be solved using finite-difference computed Jacobians, for efficiency we derived the Jacobians of the objective function and constraint functions.

The Jacobian of (17) is as follows

$$
\mathbf{J}_{0}=\frac{\partial f_{0}(\mathbf{x})}{\partial \mathbf{x}}=2 \mathbf{J}_{p p}\left(\mathbf{p}_{p}-\mathbf{p}_{t}\right)
$$

The Jacobian of (12) is

$$
\mathbf{J}_{p p}=\frac{\partial \mathbf{p}_{p}}{\partial \mathbf{x}}=\mathbf{J}_{p}+\mathbf{t} \mathbf{J}_{d}+d \mathbf{J}_{t}
$$

where

$$
\mathbf{J}_{p}=\frac{\partial \mathbf{p}}{\partial \mathbf{x}} \quad \mathbf{J}_{d}=\frac{\partial d}{\partial \mathbf{x}} \quad \mathbf{J}_{t}=\frac{\partial \mathbf{t}}{\partial \mathbf{x}}
$$

we rewrite (10) as

$$
d_{1,2}(\mathbf{x})=A \pm B
$$

and decompose the Jacobian as

$$
\begin{aligned}
\mathbf{J}_{d} & =\frac{\partial d_{i}}{\partial \mathbf{x}} \quad \text { where } i=\arg \max \left\{d_{1}, d_{2}\right\} \\
\mathbf{J}_{A} & =\frac{\partial A}{\partial \mathbf{x}}=\left(\mathbf{p}-\mathbf{p}_{s}\right)^{T} \mathbf{J}_{t}+\mathbf{t}^{T} \mathbf{J}_{p} \\
\mathbf{J}_{B} & =\frac{\partial B}{\partial \mathbf{x}}=\frac{\mathbf{J}_{A} A-\left(\mathbf{p}-\mathbf{p}_{s}\right) \mathbf{J}_{p}}{B}
\end{aligned}
$$

In order to obtain the Jacobians of the distal position and tangents, we revert to the constant curvature equations.

$$
\begin{aligned}
\frac{\partial \mathbf{p}}{\partial k} & =\frac{1}{k^{2}}\left[(\varphi \cos \varphi-\sin \varphi) \mathbf{t}_{0}+(\varphi \sin \varphi+\cos \varphi-1)\right. \\
\frac{\partial \mathbf{t}}{\partial k} & =L\left[\cos \varphi \mathbf{n}_{0}-\sin \varphi \mathbf{t}_{0}\right]
\end{aligned}
$$

The length also affects the curvature so that

$$
\frac{\partial \mathbf{p}}{\partial L}=\mathbf{t}+\frac{\partial \mathbf{p}}{\partial k} \frac{\partial k}{\partial L}
$$

In order to determine the change in curvature for a change in length, we differentiate (7) to the length and rearrange to obtain

$$
\frac{\partial k}{\partial L}=\frac{-M B_{0} k \cos (\gamma-\varphi)}{E I+M B_{0} \cos (\gamma-\varphi)}
$$

Similarly, we obtain

$$
\frac{\partial \mathbf{t}}{\partial L}=k\left[\cos \varphi \mathbf{n}_{0}-\sin \varphi \mathbf{t}_{0}\right]+\frac{\partial \mathbf{t}}{\partial k} \frac{\partial k}{\partial L}
$$

To obtain the effect of a change in the manipulation plane angle $\theta$, recall (2). Using the derivative of (3)

$$
\frac{\partial \mathbf{R}(\mathbf{v}, \theta)}{\partial \theta}=\cos \theta[\mathbf{v}]_{\times}+\sin \theta[\mathbf{v}]_{\times}^{2}
$$

we obtain

$$
\begin{aligned}
& \frac{\partial \mathbf{p}}{\partial \theta}=(1-\cos \varphi) \frac{\partial \mathbf{R}\left(\hat{\mathbf{n}}_{0}, \theta\right)}{\partial \theta} \hat{\mathbf{n}}_{0} \\
& \frac{\partial \mathbf{t}}{\partial \theta}=\sin \varphi \frac{\partial \mathbf{R}\left(\hat{\mathbf{n}}_{0}, \theta\right)}{\partial \theta} \hat{\mathbf{n}}_{0}
\end{aligned}
$$

Grouping the previous equations completes the derivation of the Jacobians.

$$
\begin{aligned}
& \mathbf{J}_{p}=\left[\begin{array}{lll}
\frac{\partial \mathbf{p}}{\partial k} & \frac{\partial \mathbf{p}}{\partial L} & \frac{\partial \mathbf{p}}{d \theta}
\end{array}\right]^{T} \\
& \mathbf{J}_{t}=\left[\begin{array}{lll}
\frac{\partial \mathbf{t}}{\partial k} & \frac{\partial \mathbf{t}}{\partial L} & \frac{\partial \mathbf{t}}{\partial \theta}
\end{array}\right]^{T}
\end{aligned}
$$

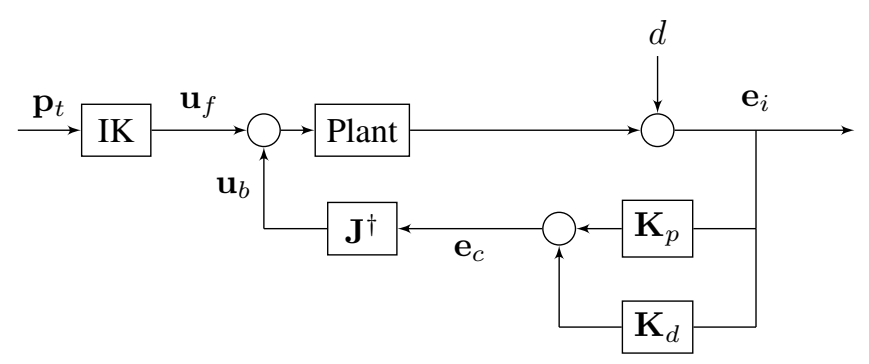

Fig. 6. Block diagram of the feedforward-feedback control strategy

\section{Closed-loop Control}

The performance of open-loop control is highly dependent on the fidelity of the model and its parameters. We, therefore, devised a feedback control scheme to correct for errors in the laser spot position. The position of the laser spot was tracked in the image as described in II-H. In practice, a low-power targeting laser such as those used in handheld endolaser tools could be used for feedback control, while the high power green laser would only be activated once the positional error is below an acceptable threshold. We used the feedforward-feedback strategy depicted in Fig. 6. 
1) Feedforward Control: The feedforward block operates in two modes. In the "inverse kinematics mode," the laser spot is rapidly controlled to the vicinity of the target using the inverse kinematics of (17). The magnetic fields associated to the inverse kinematic solution are computed using (7) and fixing the field magnitude to $B_{0}=60 \mathrm{mT}$. In the "incremental mode," which is used for moving between adjacent target points, only the length computed by the inverse kinematics is used, and the magnetic field is incremented from its previous value $\mathbf{B}_{b}[k-1]$. The output of the feedforward block is the feedforward input $\mathbf{u}_{f}$.

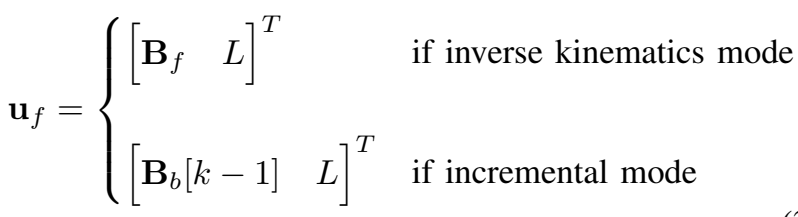

2) Feedback Control: The feedback block corrects for positional errors modelled as disturbance $d$. Given an instantaneous position error $\mathbf{e}_{i}=\mathbf{p}_{t, i}-\mathbf{p}_{p, i}$ in image coordinates, and its time first-difference $\Delta \mathbf{e}_{i}$, we used the following proportional derivative (PD) control.

$$
\Delta \mathbf{B}_{b}=\mathbf{J}^{\dagger}\left(\mathbf{K}_{p} \mathbf{e}_{i}+\mathbf{K}_{d} \Delta \mathbf{e}_{i}\right)
$$

where $\mathbf{J}^{\dagger}$ denotes the Moore-Penrose inverse of the Jacobian relating changes in the magnetic field to changes in the laser spot's position in the image. $\mathbf{K}_{p}$ and $\mathbf{K}_{d}$ are $2 \times 2$ matrices containing the gains for the PD controller. The output of the feedback block is the feedback input $\mathbf{u}_{b}$.

$$
\mathbf{u}_{b}=\left[\begin{array}{ll}
\Delta \mathbf{B}_{b} & 0
\end{array}\right]^{T}
$$

Note that the length is not modified by the feedback block since the laser spot can be moved by bending the tip, provided that the tip does not bend so much that it comes into contact with the eye. The circle of safety of III-F could be used to delimit areas in which it is safe to modify the magnetic fields using the controller.

The feedforward and feedback inputs are combined to a single input $\mathbf{u}$.

$$
\mathbf{u}=\mathbf{u}_{f}+\mathbf{u}_{b}=\left[\begin{array}{ll}
\mathbf{B} & L
\end{array}\right]^{T}
$$

The magnetic field component of the input $\mathbf{B}$ is converted to electromagnet currents using (16).

\section{RESULTS}

\section{A. Experimental Protocol}

To validate the performance of our controller in the eye phantom conditions, we generated an array of 273 target points evenly spaced by 50 pixels in the image space covering the posterior retina region. The $2 \mathrm{D}$ target points were converted to $3 \mathrm{D}$ positions using the process detailed in III-C. The average calculated distance between target points in 3D space was $950 \mu \mathrm{m}$. The laser was set to continuous operation mimicking a tracking laser on a conventional photocoagulation laser, such that it could be tracked continuously in the image. The target points were iterated horizontally, switching

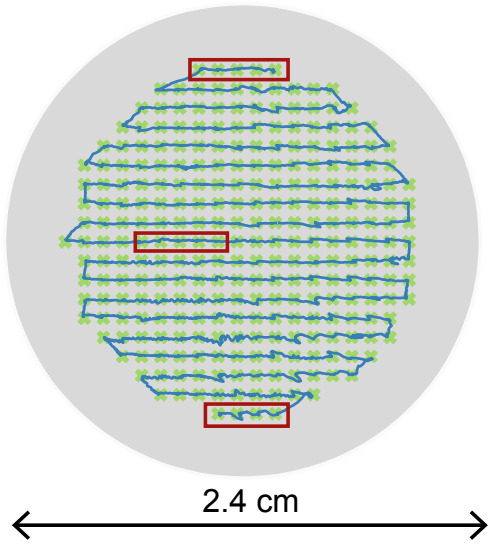

Fig. 7. 2D Representation of the tracked laser spot in the image during the experiment. The trajectory as tracked using image processing is shown as the blue line. The target points are shown as the green crosses. A representation of the eye model is shown as the gray circle. The gray circle represents the projection of the central slice of the sphere parallel to the image plane. The diameter of the sphere is $2.4 \mathrm{~cm}$. The red rectangles represent subsections of the trajectory that are represented in Fig. 8.
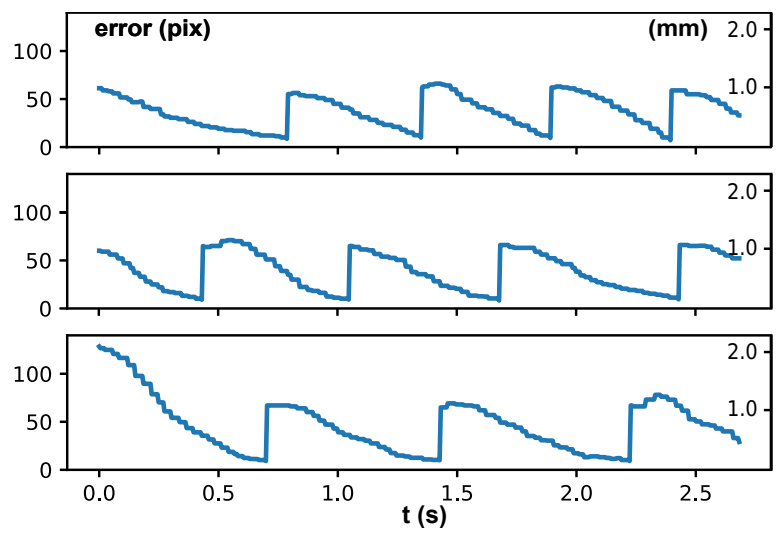

Fig. 8. Sample of the response of position error magnitude $\left\|\mathbf{e}^{i}\right\|$ over a time slice. The errors are converted to millimeters in a plane parallel to the image plane passing through the sphere center. The plots represent the top, middle, and bottom red rectangles in Fig. 7 respectively.

directions at each line to minimize a "carriage-return". The controller was given a maximum time of $1.0 \mathrm{~s}$ per target point. A target was considered reached if the pixel distance between the target and the laser spot was below 10 pixels corresponding to $160 \mu \mathrm{m}$ at the distance of the sphere's center. The Early Treatment Diabetic Treatment Research Group (ETDRS) clinical guidelines recommend a spot size of $500 \mu \mathrm{m}$, with a distance between spots of $250 \mu \mathrm{m}$ [19]. The 10 pixel threshold was determined to be sufficiently accurate given these guidelines. The trajectory followed by the laser spot is shown in Fig. 7, and three samples of positional errors over time are shown in Fig. 8.

Once having reached the target position, the controller was paused for $300 \mathrm{~ms}$ to mimic the duration of the photocoagulation, which is three times longer than the clinically suggested $100 \mathrm{~ms}$ in which the image would likely be saturated by the therapeutic laser pulse. The entire experiment took $3 \mathrm{~min} 25 \mathrm{~s}$ to complete, and a video is available in the supplementary material. 


\section{B. Discussion}

Obtaining ground truth data for such an experiment was not possible due to the difficulty of visualizing the laser spot from different angles given the space constraints of the OctoMag. Evaluation was performed using image data from the back camera. Nevertheless, image data provides substantial qualitative demonstration of control performance. The trajectory of Fig. 7 and the video in the supplementary material clearly show that all of the target points were reached with considerable accuracy (i.e within the $160 \mu \mathrm{m}$ threshold defined above).

The average amount of time between the selection of a new target and the time at which it was reached was $0.49 \mathrm{~s}$ ( $\min 0.17 \mathrm{~s} \max 0.80 \mathrm{~s})$. As can be seen in Fig. 8, there were differences in the step responses depending on the target's position on the sphere. For example, the points in the middle red rectangle have a faster step response than the points in the bottom rectangle. While no extensive reporting of the average time taken by a human operator was found in the literature, estimates from [5] of 1500 points over a session of $20 \mathrm{~min}$ roughly indicate $0.7 \mathrm{~s}$, when removing $0.1 \mathrm{~s}$ of ablation time per point. Furthermore, there is a margin for improvement in the control speed of our method, since we did not operate at the maximum bandwidth of the OctoMag. The main bottlenecks were the image framerate and image processing, which could be further optimized for performance. Additionally, for demonstration purposes, we used a less dense array of points as those used in practice. A larger number of points could be reached without changing the dynamic characteristics of our method. While it is difficult to make conclusive statements on the speed improvements without a direct comparison with a manual procedure, results from a study using the Micron showed that full automation resulted in higher speeds while also improving accuracy over manual operation [20].

We do note the following caveats in our model. The human eyeball is not perfectly spherical, rigid, and of equal diameter for all patients. The optics of the eye and the wide-angle viewing systems used in retinal surgery introduce a more complex optical model than the standard camera model used here. Nevertheless, most properties, including eye radius and lens characteristics, can be estimated from biometric data such as routine optical coherence tomography (OCT) [21], while the wide-angle viewing system and surgical microscope can be calibrated separately before an operation. An estimate for $\mathbf{T}_{c, s}$, the transformation between the eyeball and the fundus camera, can also be estimated using registration of features on the iris. The proximal insertion pose could also be registered by mounting the linear advancer to a calibrated remote-center-of motion device.

Moreover, accurate calibration may not be required for performing visual servoing as shown in this paper. We observed robustness in the control with regard to modeling inaccuracies, provided that they did not significantly alter the directions of movement of the probe in the camera image. After addressing the aforementioned differences, our method will be suitable for experiments in ex-vivo porcine eyes, as the next step towards clinical application.

\section{CONCLUSIONS}

We demonstrated a proof-of-concept system for a magnetically steered endolaser probe for performing PRP for treating diabetic retinopathy. The kinematics were modeled and used to develop closed-loop control for automation of the task. The system was demonstrated in a dry eye phantom showing qualitatively high accuracy at speeds faster than human operation.

\section{REFERENCES}

[1] R. Lee, T. Y. Wong, and C. Sabanayagam, "Epidemiology of diabetic retinopathy, diabetic macular edema and related vision loss," Eye and Vision, vol. 2, no. 1, p. 17, 2015.

[2] R. P. Singh, "Global Trends in Retina Survey," American Soc. of Retina Specialists, Tech. Rep., 2018.

[3] N. M. Bressler, R. W. Beck, and F. L. Ferris, "Panretinal Photocoagulation for Proliferative Diabetic Retinopathy," New England J. of Med., vol. 365, no. 16, pp. 1520-1526, 2011.

[4] M. Nagpal, S. Marlecha, and K. Nagpal, "Comparison of laser photocoagulation for diabetic retinopathy using 532-nm standard laser versus multispot pattern scan laser,' Retina, vol. 30, no. 3, pp. 452-458, 2010.

[5] F. Kuhn, "Endolaser," in Vitreoretinal Surgery: Strategies and Tactics, 2016, ch. 30.

[6] B. C. Becker, C. Robles Valdivieso, J. Biswas, L. A. Lobes, and C. N. Riviere, "Active guidance for laser retinal surgery with a handheld instrument," Proc. 31st Ann. Int. Conf. IEEE Eng. Med. Bio. Soc. (EMBC 2009), pp. 5587-5590, 2009.

[7] S. Yang, J. N. Martel, L. A. Lobes Jr, and C. N. Riviere, "Techniques for robot-aided intraocular surgery using monocular vision," Int. J. of Robot. Res., vol. 37, no. 8, pp. 931-952, 2018.

[8] C. Pappone, G. Vicedomini, F. Manguso, F. Gugliotta, P. Mazzone, S. Gulletta, N. Sora, S. Sala, A. Marzi, G. Augello, L. Livolsi, A. Santagostino, and V. Santinelli, "Robotic magnetic navigation for atrial fibrillation ablation," J. American College Cardiology, vol. 47, no. 7, pp. 1390-1400, 2006.

[9] L. B. Kratchman, T. L. Bruns, J. J. Abbott, and R. J. Webster, "Guiding Elastic Rods with a Robot-Manipulated Magnet for Medical Applications," IEEE Trans. Rob., vol. 33, no. 1, pp. 227-233, 2017.

[10] S. L. Charreyron, B. Zeydan, and B. J. Nelson, "Shared control of a magnetic microcatheter for vitreoretinal targeted drug delivery," in Proc. IEEE Int. Conf. Robot. Autom., 2017, pp. 4843-4848.

[11] M. P. Kummer, J. J. Abbott, B. E. Kratochvil, R. Borer, A. Sengul, and B. J. Nelson, "Octomag: An electromagnetic system for 5-DOF wireless micromanipulation," IEEE Trans. Robot., vol. 26, no. 6, pp. 1006-1017, dec 2010

[12] I. Bekerman, P. Gottlieb, and M. Vaiman, "Variations in Eyeball Diameters of the Healthy Adults," J. of Ophthalmology, vol. 2014, 2014.

[13] G. Bradski, "OpenCV Library," Dr. Dobb's Journal of Software Tools.

[14] I. Tunay, "Modeling magnetic catheters in external fields," in 26th Ann. Int. Conf. IEEE Eng. Med. Bio. Soc. (EMBC 2004), vol. 3. IEEE, 2004, pp. 2006-2009. [Online]. Available: http://ieeexplore.ieee.org/document/1403591/

[15] S. Agarwal, K. Mierle, and Others, "Ceres solver," http://ceres-solver.org.

[16] A. J. Petruska, J. Edelmann, and B. J. Nelson, "Model-Based Calibration for Magnetic Manipulation,” IEEE Trans. Magn., vol. 53, no. 7, pp. 1-1, jul 2017.

[17] D. Kraft, "A software package for sequential quadratic programming," 1988 , p. 33.

[18] E. Jones, T. Oliphant, P. Peterson, et al., "SciPy: Open source scientific tools for Python," 2001.

[19] "Early Photocoagulation for Diabetic Retinopathy: ETDRS Report Number 9," Ophthalmology, vol. 98, no. 5, pp. 766-785, 1991.

[20] S. Yang, R. A. MacLachlan, J. N. Martel, L. A. Lobes, and C. N. Riviere, "Comparative Evaluation of Handheld Robot-Aided Intraocular Laser Surgery," IEEE Trans. Rob., vol. 32, no. 1, pp. 246-251, 2016.

[21] C. Bergeles, K. Shamaei, J. J. Abbott, and B. J. Nelson, "Single-camera focus-based localization of intraocular devices," IEEE Trans. Biomed. Eng., vol. 57, no. 8, pp. 2064-2074, 2010. 